\title{
Factors Associated with Development of High-Grade Squamous Intraepithelial Lesions of the Uterine Cervix in Women Younger than 30 Years
}

\author{
Jongpeeti Wudtisan, Charuwan Tantipalakorn, Kittipat Charoenkwan, \\ Rung-Aroon Sreshthaputra, Jatupol Srisomboon*
}

\begin{abstract}
Objective: To determine the factors associated with the increased risk of developing high-grade squamous intraepithelial lesions (HSIL) of the uterine cervix in women younger than 30 years compared with those aged $\geq 30$ years who also had HSIL. Methods: Patients with HSIL who underwent loop electrosurgical excision procedure (LEEP) between January 2006 and July 2017 at Chiang Mai University Hospital were retrospectively reviewed. We analyzed the factors associated with the development of HSIL by comparing two age groups between women aged $<30$ years and those aged $\geq 30$ years. The factors analyzed included the well-recognized risk factors for cervical cancer, i.e. age at sexual debut, number of sexual partners, use of oral contraceptive (OC) pills, smoking history, sexually transmitted diseases and HIV status. Univariate and multivariate logistic regressions were used to assess factors associated with the increased risk of developing HSIL in women younger than 30 years compared with those aged $\geq 30$ years. Results: During the study period, there were 345 patients with HSIL, 30 were $<30$ years (case group) and 315 aged $\geq 30$ years (control group). By multivariate analyses, early sexual debut(OR, 2.86; 95\% CI, 1.01-8.13; $\mathrm{P}=0.047$ ), multiple sexual partners (OR, 2.94; 95\% CI, 1.23-7.02; $\mathrm{P}=0.015)$, history of genital warts $(\mathrm{OR}, 20.46 ; 95 \% \mathrm{CI}, 2.27-183.72 ; \mathrm{P}=0.007)$ and history of smoking $(\mathrm{OR}, 2.95 ; 95 \% \mathrm{CI}, 1.10-7.93 ; \mathrm{P}=0.032)$ were significantly associated with the development of HSIL in women younger than 30 years when compared with those aged $\geq 30$ years. The OC use, HIV status and underlying diseases were not significantly different in both groups. Conclusion: Early age at sexual debut, multiple sexual partners, history of genital warts and smoking are significant risk factors for developing HSIL in women younger than 30 years. Cervical cancer screening should be considered in young women with such factors.
\end{abstract}

Keywords: High-grade squamous intraepithelial lesions- risk factors- younger women - uterine cervix

Asian Pac J Cancer Prev, 20 (4), 1031-1036

\section{Introduction}

Cervical cancer is the second most commonly diagnosed cancer after breast cancer and the third leading cause of cancer-related death in developing countries. In Thailand, cervical cancer is the second most common cancer in women with an estimated 10,000 new cases and 5,200 deaths in 2012 (Torre et al., 2015). From the report of Public Health Ministry of Thailand in 2015, the age-standardized incidence rate of cervical cancer in Thailand and Chiang Mai are 14.4 and 17.4 per 100,000 women per year, respectively (Imsamran et al., 2015).

The most important determinants for development of cervical cancer is human papillomavirus (HPV) infection especially the high-risk strains, type 16 and 18 which cause approximately $70 \%$ of cervical cancer and $50 \%$ of its precursors (Kahn, 2009). Factors that increase the risk of developing cervical cancer are those that increase the exposure to HPV infection or facilitate HPV persistence, i.e. early onset of sexual activity, multiple sexual partners, high parity, low immunocompetence, oral contraceptive use and cigarette smoking (Appleby et al., 2007; Cancer, 2007; Klumb et al., 2010; Ajah et al., 2014). A cross-sectional study showed that age at sexual debut of 9 - 15 years increased the risk of HPV infection (Giuliano et al., 1999). The number of sexual partners more than 5 in a life time is independently associated with cervical HPV infection with an odds ratio of 3.5 (Remschmidt et al., 2013).

HPV infection is highly prevalent in sexually active women particularly in young women. However, most HPV infections in younger women spontaneously clear within 8 - 24 months (Ho et al., 1998). Although the prevalence of cervical HPV infection decreases after the age of 30

Department of Obstetrics and Gynecology, Faculty of Medicine, Chiang Mai University, Chiang Mai, Thailand. *For Correspondence: jatupol1957@hotmail.com 
years, however, the risk of HPV persistence increases with age. Development of precancerous cervical lesions before the age of 30 years is quite uncommon. Cervical cancer screening is accordingly, recommended in women after the age of 30 years with the aim to detect precancerous lesion, i.e. high-grade squamous intraepithelial lesions (HSIL) that can be treated before progressing to cervical cancer.

In 2013, World Health Organization (WHO) has recommended to start screening with Pap cytology or visual Inspection with acetic acid (VIA) in women 30 years of age and older because of their higher risk of cervical cancer. In women who test negative on VIA or cytology, the screening interval for repeat screening should be every 3-5 years (WHO, 2013). In Thailand, the Ministry of Public Health has recommended to start cervical cancer screening with Pap cytology in women aged 30 years at 5 years interval until the age of 60 years (Wilailak and Lertchaipattanakul, 2016). However, there is a proportion of women who develop HSIL before the age of 30 years. We were interested in exploring what factors increase the risk of developing HSIL in women younger than 30 years in order to identify women at risk to have earlier screening before the age of recommendation. Accordingly, this study was conducted to evaluate the factors associated with the increased risk of developing HSIL in women aged less than 30 years compared with those aged $\geq 30$ years who also had HSIL.

\section{Materials and Methods}

After approval of the Research Ethics Committee of Chiang Mai University Hospital, women who underwent loop electrosurgical excision procedure (LEEP) for pathological HSIL of the uterine cervix at Chiang Mai University Hospital between January 1, 2006 and July 31, 2017 were retrospectively reviewed. The data resources for review were medical records of the colposcopy clinic of Obstetrics and Gynecology Department and the computerized database of Chiang Mai University Hospital. The patient characteristics and treatment details were reviewed, including the cervical cancer risk factors, i.e. age at first sexual relation, number of sexual partners, parity, current use of contraception either oral pills or non-oral contraception, history of smoking, HIV status, history of sexually transmitted diseases (STD) and underlying diseases. Multiple sexual partners was defined as number of partners $\geq 2$.

The inclusion criteria were the women who had pathological diagnosis of HSIL obtained from LEEP. The women who had history of gynecologic cancer or hysterectomy for malignant gynecologic disease, incomplete medical record and pregnancy were excluded. The main outcome was to determine the risk factors associated with the development of HSIL in women aged less than 30 years compared with those aged 30 years or more. The descriptive data were presented as percentage / range or means $+\mathrm{SD}$, as appropriate. The statistical analysis was performed using SPSS version 21.0 (IBM Corp. Released 2012; IBM SPSS Statistics for Windows, Version 21.0. Armonk, NY: USA). Univariate analysis was performed to examine the association between clinical risk factors and development of HSIL. Relevant odd ratios $(\mathrm{OR})$ and $95 \%$ confidence intervals $(95 \% \mathrm{CI})$ were calculated. The $\mathrm{p}$-value $<0.05$ was considered significant. The factors with the $\mathrm{p}$-value of $<0.05$ from the univariate analysis were further evaluated in multivariate analysis.

\section{Results}

During the study period, 781 patients underwent LEEP for HSIL of the uterine cervix

$64(8.2 \%)$ were younger than 30 years. Of these, 436 patients were excluded due to incomplete medical records (385), pregnancy (14) and history of gynecologic malignancy (37) as shown in Figure 1. Finally, a total of 345 patients were enrolled in this study. Among the 345

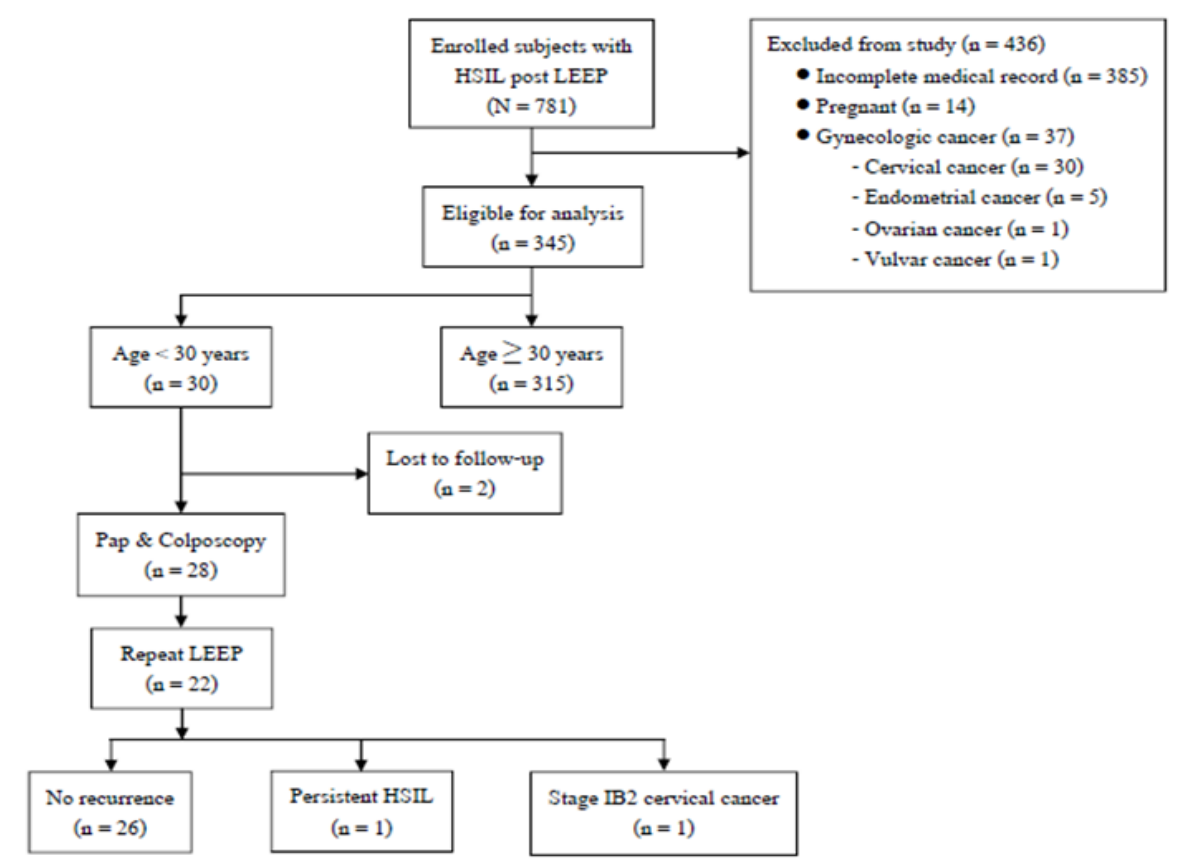

Figure 1. Flowchart of the Study Population 
Table 1. Patient Characteristics and Univariate Analysis for Risk of Developing HSIL in Women Aged $<30$ Years versus Women Aged $\geq 30$ Years

\begin{tabular}{|c|c|c|c|}
\hline \multirow[t]{2}{*}{ Risk factors } & \multicolumn{2}{|c|}{ Number/Total (\%) } & \multirow[t]{2}{*}{ P-value } \\
\hline & Case (age $<30$ years) & Control (age $\geq 30$ years) & \\
\hline Age at sexual debut & & & 0.006 \\
\hline$<21 \mathrm{yr}$ & $25 / 30(83.3 \%)$ & $181 / 315(57.5 \%)$ & \\
\hline$\geq 21 \mathrm{yr}$ & $5 / 30(16.7 \%)$ & $134 / 315(42.5 \%)$ & \\
\hline Number of sexual partners & & & 0.001 \\
\hline 1 & $10 / 30(33.3 \%)$ & $109 / 315(34.6 \%)$ & \\
\hline$\geq 2$ & $20 / 30(66.7 \%)$ & $206 / 315(65.4 \%)$ & \\
\hline Current contraception & & & 0.311 \\
\hline Oral contraceptives & $9 / 30(30 \%)$ & $69 / 315(21.9 \%)$ & \\
\hline Non-oral contraceptives & $21 / 30(70 \%)$ & $246 / 315(78.1 \%)$ & \\
\hline HIV status & & & 0.978 \\
\hline Negative & $27 / 30(90 \%)$ & $284 / 315(90.2 \%)$ & \\
\hline Positive & $3 / 30(10 \%)$ & $31 / 315(9.8 \%)$ & \\
\hline History of genital warts & & & $<0.001$ \\
\hline Yes & $3 / 29(10.3 \%)$ & $2 / 312(0.6 \%)$ & \\
\hline No & $26 / 29(89.7 \%)$ & $310 / 312(99.4 \%)$ & \\
\hline History of smoking & & & 0.001 \\
\hline Yes & $8 / 30(26.7 \%)$ & $26 / 315(8.3 \%)$ & \\
\hline No & $22 / 30(73.3 \%)$ & $289 / 315(91.7 \%)$ & \\
\hline Underlying disease & & & 0.25 \\
\hline Yes & $7 / 30(23.3 \%)$ & $105 / 312(33.7 \%)$ & \\
\hline No & $23 / 30(76.7 \%)$ & $207 / 312(66.3 \%)$ & \\
\hline
\end{tabular}

HIV, Human immunodeficiency virus

Table 2. Multivariate Analysis for Risk of Developing HSIL in Women Aged $<30$ Years versus Women Aged $\geq 30$ Years

\begin{tabular}{lcc}
\hline Risk factors & OR $(95 \% \mathrm{CI})$ & P value \\
\hline Early age at sexual debut & $2.86(1.01-8.13)$ & 0.047 \\
Multiple sexual partners ${ }^{1}$ & $2.94(1.23-7.02)$ & 0.015 \\
History of genital warts & $20.46(2.27-183.72)$ & 0.007 \\
History of smoking & $2.95(1.10-7.93)$ & 0.032 \\
\hline${ }^{1}$, Numbers of partners $\geq 2$ & &
\end{tabular}

patients with HSIL, 30 were younger than 30 years (case group) and 315 aged $\geq 30$ years (control group). The mean ages of the patients in the case and the control groups were 26.5 years (range: $16-29$ years) and 48.3 years (range: 31-79 years), respectively. The mean life-time sexual partners was 2.6(range: 1-10) in the case group and was 1.5 (range:1-6) in the control group. The mean ages at first sexual activity in the case and the control groups were 18.7 years (range: $13-29$ years) and 21.1 years (13-35 years), respectively.

The patient characteristics of both groups and univariate analysis for risk factors associated with the development of HSIL are shown in Table 1. The case group, when compared with control group had significant difference in the factors of early sexual debut $(<21$ years), multiple sexual partners $(\geq 2)$, history of genital warts and cigarette smoking. In multivariate analysis, early sexual debut (OR, 2.86; 95\% CI, 1.01-8.13; $\mathrm{P}=0.047$ ), multiple sexual partners (OR, 2.94; 95\% CI, 1.23-7.02; $\mathrm{P}=0.015)$, history of genital warts (OR, 20.46; 95\% CI, 2.27-183.72; $\mathrm{P}=0.007)$ and history of smoking ( $\mathrm{OR}, 2.95$; $95 \% \mathrm{CI}, 1.10-7.93 ; \mathrm{P}=0.032$ ) were significantly associated with the development of HSIL in women younger than 30 years (Table 2). Oral contraceptive use, HIV status and underlying diseases were not significantly different in both groups.

After LEEP in the 30 patients, 2 were lost to follow up. The remaining 28 patients were followed with Pap cytology and colposcopy at 6-months interval. Of these, 22 patients underwent repeat LEEP for abnormal cervical cytology and 18 had HSIL and 3 had LSIL. The remaining 1 had stage IB2 cervical cancer and were treated with concurrent chemoradiation. The last visits of the 28 patients showed that 26 had no recurrence, 1 had persistent HSIL and underwent simple hysterectomy, the remaining 1 with stage IB2 cervical cancer was under follow-up after chemoradiation.

\section{Discussion}

Our study showed that among women with a diagnosis of HSIL of the uterine cervix, the significant risk factors of developing these lesions in women younger than 30 years when compared with those aged $\geq 30$ years are early sexual debut, multiple sexual partners, history of genital warts and smoking. Numerous factors have been recognized as increased risk for cervical cancer including 
young age at first sexual intercourse, cigarette smoking, multiple lifetime sexual partners, high parity, increasing duration of contraceptive pill use, younger age at first full-term pregnancy, history of genital warts, low socioeconomic status and immunosuppression (Giuliano et al., 1999; Cancer, 2007; Remschmidt et al., 2013; Zhu et al., 2015). In fact, these factors indicate the increased risk of exposure to oncogenic HPV infection or having compromised immune response to HPV infection, i.e. multiple sexual partners, young age at sexual debut, cigarette smoking, immunosuppression and previous treatment for genital warts. HPV infection was highly prevalent in women aged under 30 years (Stuardo et al., 2012). Women with history of high-risk HPV infection had an increased risk of developing HSIL in the future (Giuliano et al., 2004).

We were interested in exploring the factors influencing the development of HSIL in women younger than 30 years comparing with those aged $\geq 30$ years because these young women were not included in the recommendation for routine cervical cancer screening in Thailand. We did not aim to compare the factors with women of the same age group who did not have HSIL since these factors have already been recognized as risk factors for cervical cancer. In the total of 781 women who were diagnosed with HSIL of the uterine cervix in the present study, only $64(8.2 \%)$ were younger than 30 years. The number of HSIL cases in younger women may be underestimated since most were detected from opportunistic screening. In the ATHENA study enrolling 42,209 women aged $\geq 25$ years in the U.S. to evaluate the performance of HPV testing as the primary screening for cervical cancer, pathological HSIL was detected in 587 women, of whom $210(35.8 \%)$ occurred in women aged 25-29 years, despite accounting for only $16.3 \%$ of all study population. Of interest, among 347 women with CIN3 or more severe lesions, 119 (34.3\%) were younger than 30 years (Wright et al., 2015). Such findings that over one-third of precancerous cervical lesions occurred in young women imply that these lesions can be detected if young women at risk are identified and appropriately screened.

In our study, age at first sexual relation of less than 21 years was significantly associated with the development of HSIL in women aged less than 30 years. The mean ages at first sexual activity seemed to be lower in younger women with HSIL (18.7 years) compared with that in older women (21.2 years) who also had HSIL. Although early age of sexual activity increases the risk of HPV acquisition, previous study noted that the age at first sexual intercourse of less than 17 year was not associated with developing HSIL in women aged 20-31 years (Remschmidt et al., 2013).

Among the women with HSIL, the number of sexual partners $\geq 2$ was significantly associated with the development of HSIL in the present study. The mean life-time sexual partners in younger women (2.6) appeared to be higher than that in the older group (1.5). This is also consistent with the previous report noting that more than 5 sexual partners was significantly correlated with HSIL in women age 20-31 years with the odds ratio (OR) of 5.1
(95\% CI, 2.1-12.5; $\mathrm{P}<0.001)$ (Remschmidt et al., 2013). The number of sexual partners more than 5 were also remarkably linked to high-risk HPV infection (Giuliano et al., 1999). It can be concluded that the increase in number of sexual partners reflected the increased probability of acquiring HPV infection.

History of sexually transmitted infections, e.g. Chlamydia trachomatis and genital herpes is epidemiologically recognized as co-factors of cervical carcinogenesis. This factor just reflects the higher chance of acquiring other genital infections especially HPV infection including genital warts. Our multivariate analysis showed that history of genital warts were significantly associated with the development of HSIL in women younger than 30 years with the OR of 20.46 (95\% CI, 2.27-183.72; $\mathrm{P}=0.007)$ when compared with women aged $\geq 30$ years. This finding corresponds with the previous report that genital warts may be epidemiological co-factors in the development of HSIL (OR, 5.7; 95\% CI, 1.2-27.6; $\mathrm{P}=0.03$ ) (Remschmidt et al., 2013).

Smoking was significantly correlated with development of HSIL in younger women when compared with the older group in our study. Previous study showed that current smoking was associated with a significantly increased risk of squamous cell carcinoma but not adenocarcinoma (Cancer, 2007). On the contrary, other studies noted that current smoking was not associated with HSIL in women aged 20-31 years (Remschmidt et al., 2013) and was not significantly associated with the occurrence of abnormal cytology (Stuardo et al., 2012).

The use of oral contraceptives (OC) was not associated with a significant increase in the risk of developing HSIL in younger women comparing with the older ones in the present study. However, the data on the duration of OC use were not available. The correlation of HSIL and cervical cancer with the OC use is not entirely consistent due to heterogeneity of the studies. The OC use of less than 5 years was not related to the risk of cervical cancer (OR, 0.77; 95\% CI,0.46-1.29) but it increased significantly for 5-9 years of use (OR , 2.72; 95\% CI,1.36- 5.46) and for $\geq 10$ years of use (OR,4.48; 95\% CI,2.24-9.36) (Bosch and de Sanjose, 2007). Another study noted that the relative risk of cervical cancer increased in the current users of $\mathrm{OC}$ and declined after the cessation of OC use (Appleby et al., 2007). Since persistent HPV infection is essential for cervical carcinogenesis, thus, the association between OC use and the risk of cervical cancer should be studied in the relevant population, i.e. HPV-positive women. A pooled data of multicenter case- control study assessing the correlation of OC use and cervical cancer in HPV-positive women noted that ever use of OC was not significantly associated with an increase in cervical cancer (OR, 1.29; CI, 0.88-1.91). However, duration of OC use was significantly associated with the development of cervical cancer, i.e. HPV-positive women who used OC for 5 to 9 years (OR, 2.82; CI, 1.46-5.42) and $\geq 10$ years (OR, 4.03; CI, 2.09-8.02) had a significant increase in the risk of cervical cancer compared with never users. The risk did not increase in women who used OC for less than 5 years (Moreno et al., 2002). 
HIV status was not significantly associated with the development of HSIL in women younger than 30 years when compared with the older ones in this study. This may be caused by small number of cases in the younger group. In general, the risk of HPV infection is higher in HIV-positive women than in women who are HIVnegative. In a study involving $1778 \mathrm{HIV}$-positive and $500 \mathrm{HIV}$-negative women, it was found that $63 \%$ of the HIV-positive women tested positive to HPV DNA while only $30 \%$ of the HIV-negative participants tested positive (Palefsky et al., 1999). HIV-positive women have been reported to have 7 times more incidence of cervical cancer than their HIV-negative counterparts (Abraham et al., 2013).

The strength of the present study was the inclusion of patients who were treated at a single institution and all pathologic specimens were examined by expert gynecologic pathologists. However, certain limitations exist. As the design of this study was retrospective, some epidemiologic data were no available, e.g. the data of sexual partners (partners with multiple sexual partner, circumcision or known HPV infection), socioeconomic status, results of the HPV testing and the patients' body mass index. Secondly, this study contained a relatively small sample size due to the rarity of this disease in young women. In addition, cervical cancer screening in women younger than 30 years in Thailand is not routinely recommended in the national policy. Therefore, younger women with precancerous cervical lesions may not be detected. Clinical implication of the findings in this study is that cervical cancer screening should also be provided to women younger than 30 years who have multiple sexual partners, early sexual debut, history of genital warts or smoking although they are not included in the routine screening program.

In conclusion, among women with pathological diagnosis of HSIL, early sexual debut, multiple sexual partners, history of genital warts and smoking are significant risk factors for developing HSIL in women younger than 30 years. Cervical cancer screening should be considered in young women with such factors.

\section{Conflict of Interest}

The authors have no conflict of interest to disclose.

\section{Acknowledgments}

The authors wish to thank Faculty of Medicine Research Fund, Chiang Mai University, for the financial support in this study.

\section{References}

Abraham AG, D’Souza G, Jing Y, et al (2013). Invasive cervical cancer risk among HIV-infected women: a North American multicohort collaboration prospective study. J Acquir Immune Defic Syndr, 62, 405-13.

Ajah L, Chigbu C, Onah H, et al (2014). Cytologic surveillance versus immediate colposcopy for women with a cervical smear diagnosis of low-grade squamous intraepithelial lesion in a poor setting in Nigeria. Onco Targets Ther, 7, 2169-73.
Appleby P, Beral V, Berrington de Gonzalez A, et al (2007). Cervical cancer and hormonal contraceptives: collaborative reanalysis of individual data for 16,573 women with cervical cancer and 35,509 women without cervical cancer from 24 epidemiological studies. Lancet, 370, 1609-21.

Bosch FX, de Sanjose S (2007). The epidemiology of human papillomavirus infection and cervical cancer. Dis Markers, 23, 213-27.

Cancer ICoESoC (2007). Comparison of risk factors for invasive squamous cell carcinoma and adenocarcinoma of the cervix: collaborative reanalysis of individual data on 8,097 women with squamous cell carcinoma and 1,374 women with adenocarcinoma from 12 epidemiological studies. Int $J$ Cancer, 120, 885-91.

Giuliano AR, Papenfuss M, De Galaz EM, et al (2004). Risk factors for squamous intraepithelial lesions (SIL) of the cervix among women residing at the US-Mexico border. Int $J$ Cancer, 109, 112-8.

Giuliano AR, Papenfuss M, Schneider A, et al (1999). Risk factors for high-risk type human papillomavirus infection among Mexican-American women. Cancer Epidemiol Biomarkers Prev, 8, 615-20.

Ho GY, Bierman R, Beardsley L, et al (1998). Natural history of cervicovaginal papillomavirus infection in young women. $N$ Engl J Med, 338, 423-8.

Imsamran W CA, Wiangnon S, Pongnikorn D, et al (2015). Cancer in Thailand. Vol. VIII, 2010-2012. Bangkok: National Cancer Institute Thailand; 2015.

Kahn JA (2009). HPV vaccination for the prevention of cervical intraepithelial neoplasia. $N$ Engl J Med, 361, 271-8.

Klumb EM, Araujo ML Jr, Jesus GR, et al (2010). Is higher prevalence of cervical intraepithelial neoplasia in women with lupus due to immunosuppression?. J Clin Rheumatol, 16, 153-7.

Moreno V, Bosch FX, Munoz N, et al (2002). Effect of oral contraceptives on risk of cervical cancer in women with human papillomavirus infection: the IARC multicentric case-control study. Lancet, 359, 1085-92.

Palefsky JM, Minkoff H, Kalish LA, et al (1999). Cervicovaginal human papillomavirus infection in human immunodeficiency virus-1 (HIV)-positive and high-risk HIV-negative women. J Natl Cancer Inst, 91, 226-36.

Remschmidt C, Kaufmann AM, Hagemann I, et al (2013). Risk factors for cervical human papillomavirus infection and high-grade intraepithelial lesion in women aged 20 to 31 years in Germany. Int J Gynecol Cancer, 23, 519-26.

Stuardo V, Agusti C, Godinez JM, et al (2012). Human papillomavirus infection in HIV-1 infected women in Catalonia (Spain): implications for prevention of cervical cancer. PLoS One, 7, e47755.

Torre LA, Bray F, Siegel RL, et al (2015). Global cancer statistics, 2012. CA Cancer J Clin, 65, 87-108.

WHO (2013). WHO Guidelines Approved by the Guidelines Review Committee. In 'WHO Guidelines for Screening and Treatment of Precancerous Lesions for Cervical Cancer Prevention', Eds World Health Organization. Copyright (c) World Health Organization 2013., Geneva.

Wilailak S, Lertchaipattanakul N (2016). The epidemiologic status of gynecologic cancer in Thailand. J Gynecol Oncol, 27, e65.

Wright TC, Stoler MH, Behrens CM, et al (2015). Primary cervical cancer screening with human papillomavirus: end of study results from the ATHENA study using HPV as the first-line screening test. Gynecol Oncol, 136, 189-97.

Zhu M, He Y, Baak JP, et al (2015). Factors that influence persistence or recurrence of high-grade squamous intraepithelial lesion with positive margins after the loop 
Jongpeeti Wudtisan et al

electrosurgical excision procedure: a retrospective study. BMC Cancer, 15, 744.

\section{(c) (i) (2)}

This work is licensed under a Creative Commons AttributionNon Commercial 4.0 International License. 